\title{
Run-up Flow of Oldroyd-B Fluid through a Parallel plate channel
}

\author{
M.VeeraKrishna ${ }^{1 *}$ and Syed Yedulla Qadri ${ }^{2}$ \\ Department of Mathematics, Rayalaseema University, Kurnool, Andhra Pradesh India 518007 \\ (* - Corresponding author)
}

\begin{abstract}
In this paper, we consider the unsteady hydrodynamic flow of an incompressible Oldroyd-B fluid in a parallel plate channel, initially induced by a constant pressure gradient. The pressure gradient is suddenly withdrawn and the upper plate moves with a uniform velocity while the lower plate continues to be at rest. The arising flow is referred to as run up flow. The unsteady governing equations are solved as initial value problem using Laplace transform technique. The expressions for velocity, shear stresses on both plates and discharge are obtained. The behaviour of the velocity, shear stresses and mass flux has been discussed in detail with respect to variations in different governing flow parameters and is presented through graphs.
\end{abstract}

Keywords: Run-up flow, Oldroyd-B fluid, Laplace transforms and parallel plate channels.

\section{Introduction}

The study of run-up flows is gaining importance due to its wide applications in different technologies. This phenomenon arises in petrochemical engineering, lubrication technology, irrigation systems, water supply and bio-fluid mechanics where the pressure gradient is suddenly withdrawn from the steady state flow which henceforth gains unsteadiness due to extraneous influence. In some technological problems, the fluid flow experiences phenomenon viz. run-up which arises due to sudden withdrawal of the pressure gradient causing the flow while its boundaries instantaneously move from rest. Under this phenomenon, steady flow in the unperturbed state gains unsteadiness later. Many research workers have paid attention to words the study of non-Newtonian fluids. In lubrication theory and in many physical situations where we come across slip flows, there arises a class of problems referred to as "run up and spin up flows". The growing importance of the use of non-Newtonian fluids in modern technology and industries has led various researchers to attempt diverse flow problems related to several non-Newtonian fluids. One such fluid that has attracted the attention of research workers in fluid mechanics during the last few decades is the Oldroyd-B fluid. This theory has several industrial and scientific applications as well, which comprise pumping fluids such as synthetic fluids, polymer thickened oils, liquid crystal, animal blood, synovial fluid present in synovial joints and the theory of lubrication (Naduvinamani et al. [1-5], Lin and Hung [6]). Kazakia and Rivlin [7] initiated the study of these flows and later Rivlin [8-10] elaborately studied the run-up and spin-up flows of viscoelastic fluids between rigid parallel plates and in circular geometries. Ramacharyulu and Raju [11] investigated the run-up flow of a viscous incompressible fluid in a long circular cylinder of porous material. Ramakrishna [12] discussed the run up and spin up flows related to a dusty viscous fluid. Later Devakar and Iyengar [14] examined the run-up flow of an incompressible couple stress fluid between two infinite rigid parallel plates. The flow was assumed to be initially induced by a constant pressure gradient between two infinite rigid parallel plates. After the steady state was attained, the pressure gradient was suddenly withdrawn and the parallel plates are set to move instantaneously with different velocities in the direction of the applied pressure gradient. The time dependence of the resultant flow was investigated. Sugunamma et al. [15] analyze the start-up flow of an incompressible Visco-elastic Rivlin-Ericksen fluid. The initial flow is assumed due to the movement of boundaries. At an instant of time t, the boundaries are suddenly brought to rest and the flow is maintained due to a prescribed pressure gradient. Veera Krishna et al. [16] discussed the hall current effects on unsteady MHD flow of rotating Maxwell fluid through a porous medium in a parallel plate channel. Raji Reddy and Sambasiva Rao [17] analyzed run-up flow of viscous incompressible fluid through a rectangular pipe, a pipe of equilateral triangular cross section, parallel plate channel and a cylinder. They solved the solved using ADI numerical technique. Raji Reddy [18] discussed with the numerical solution of the run-up flow of a viscous incompressible fluid through a pipe whose cross-section is an equilateral triangle. The problem is solved numerically, using a five-point formula. From this, it is observed that Reynold number has a great influence on the run-up flows. If it is large, the fluid comes to rest after a long time. Mahaboob Basha [19] discussed Visco-elastic fluid flow and Heat Transfer through porous medium where he has studied by considering visco-elastic RivlinEricksen fluid between parallel plates subjected to a constant suction. Malleswari [20] discussed run-up flow of Rivlin-Ericksen fluid with porous lining. Devakar and Iyengar [21] studied the run up flow of an incompressible micropolar fluid between two horizontal infinitely long parallel plates. Initially a flow of the fluid is induced by a constant pressure gradient until steady state is reached. After the steady state is reached, the pressure gradient is suddenly withdrawn while the two plates are impulsively started with different velocities in their own plane. Using 
the Laplace transform technique and adopting the state space approach, obtained the velocity and micro rotation components in Laplace transform domain. In this paper, we have considered the unsteady magneto hydro dynamic flow of an incompressible Oldroyd-B fluid in a parallel plate channel, initially induced by a constant pressure gradient. The pressure gradient is suddenly withdrawn and the upper plate moves with a uniform velocity while the lower plate continues to be at rest. The arising flow is referred to as run up flow. The unsteady governing equations are solved as initial value problem using Laplace transform technique.

Oldroyd-B Model: The Oldroyd-B model is a simple form of the more elaborate and rarely used Oldroyd 8constant model which also contains the upper convected, the lower convected, and the co-rotational Maxwell equations as special cases. Oldroyd-B is the second simplest nonlinear visco-elastic model and is apparently the most popular in visco-elastic flow modeling and simulation. It is the nonlinear equivalent of the linear Jeffreys model, as it takes account of frame invariance in the nonlinear regime. Oldroyd-B model can be obtained by replacing the partial time derivatives in the differential form of the Jeffreys model with the upper convected time derivatives.

$$
\tau+\lambda_{1} \stackrel{\nabla}{\tau}=\mu_{0}\left(\dot{\gamma}+\lambda_{2} \dot{\gamma}\right)
$$

Where $\dot{\gamma}$ is the upper convected time derivative of the rate of strain tensor given by

$$
\dot{\gamma}=\frac{\partial \dot{\gamma}}{\partial t}+V \cdot \nabla \dot{\gamma}-(\nabla V)^{T} \cdot \dot{\gamma}-\dot{\gamma} \cdot \nabla V
$$

where $\tau$ is the extra stress tensor, $V$ is the fluid velocity vector, $\lambda_{1}$ and $\lambda_{2}$ are the fluid relaxation and retardation times respectively, $t$ is the time, $\mu_{0}$ is the low shear viscosity and $\dot{\gamma}$ is the rate of strain tensor.

\section{Formulation and Solution of the problem}

We consider the flow of an incompressible Oldroyd-B fluid between two infinite rigid parallel plates $y=0$ and $y=h$ along the direction of $x$-axis (Fig.1). Since the flow is along the $x$-direction, we take the velocity $q=(u(y, t), 0,0)$, which satisfies the continuity equation.

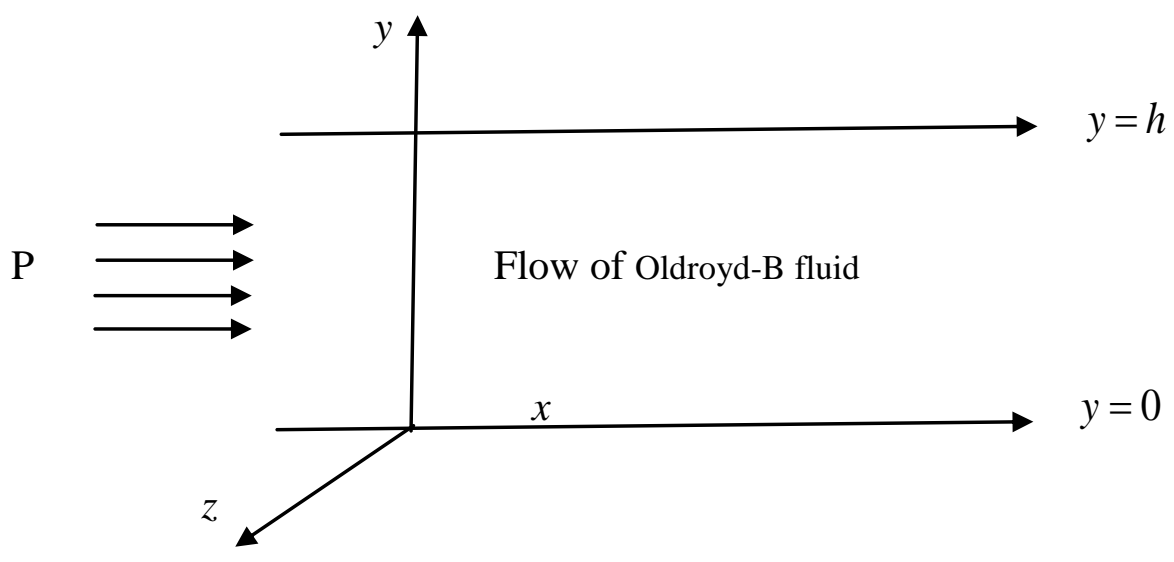

Fig 1. Physical Configuration of the problem

We consider a Cartesian system $\mathrm{O}(x, y)$ so that the fluid flow takes place within boundary plates $y=0$ and $y=h$. The linear momentum equation governing the flow $u(y, t)$ is given by

$$
\lambda\left(\frac{\partial^{2} u}{\partial t^{2}}\right)+\frac{\partial u}{\partial t}=-\frac{1}{\rho}\left(\frac{\partial p}{\partial x}+\lambda \frac{\partial^{2} p}{\partial t \partial x}\right)+v\left(\frac{\partial^{2} u}{\partial y^{2}}+\lambda_{r} \frac{\partial^{3} u}{\partial t \partial y^{2}}\right)
$$

Where $\rho$ the density, $p$ is the pressure, $\mu$ is the coefficient of viscosity and $\lambda, \lambda_{r}$ are the relaxation and retardation times.

The boundary conditions are

$u=\left\{\begin{array}{l}U ; y=h \\ 0 ; y=0\end{array} \quad(t \geq 0)\right.$ 
We consider the run-up flow of an Oldroyd-B fluid through the parallel plate channel. Initially the flow due to a prescribed pressure gradient with boundaries at rest and at the time $t>0$, the pressure gradient is withdrawn and the upper plate moves with a uniform velocity while the lower plate continues to be at rest.

The equation governing the initial flow is

$v\left(\frac{\partial^{2} u}{\partial y^{2}}\right)=\frac{1}{\rho}\left(\frac{\partial p}{\partial x}\right)$

The initial conditions are

$u=\left\{\begin{array}{l}0 ; y=h \\ 0 ; y=0\end{array}(t \leq 0)\right.$

We introduce the non-dimensional variables

$x^{*}=\frac{x}{h}, y^{*}=\frac{y}{h}, u^{*}=\frac{u}{U}, p^{*}=\frac{p}{\rho U^{2}}, t^{*}=\frac{t U}{h}$

Using non-dimensional variables, the governing equations are (dropping the asterisk)

$\lambda_{1}\left(\frac{\partial^{2} u}{\partial t^{2}}\right)+\frac{\partial u}{\partial t}=\frac{1}{R}\left(\frac{\partial^{2} u}{\partial y^{2}}\right)+\lambda_{2}\left(\frac{\partial^{3} u}{\partial t \partial y^{2}}\right)$

$\frac{\partial^{2} u}{\partial y^{2}}=P R$

Where, $\quad R=\frac{\rho U h}{\mu}$ Reynolds's number, $\lambda_{1}=\frac{\lambda U}{h}$ and $\lambda_{2}=\frac{\lambda_{r} U}{\rho h^{2}}$ are material parameters related to relaxation and retardation respectively (Oldroyd-B fluid parameters) and $P=\frac{\partial p}{\partial x}$ is the applied pressure gradient.

Using the boundary conditions (2.4), the equation (2.6) reduces to

$u=\frac{P R}{2} y(y-1)$

Applying the Laplace transforms (Honig et al. [13]) to the equation (2.5) and using the equation (2.7), we get

$\bar{u}(y, s)=A \operatorname{Cosh}(a y)+B \operatorname{Sinh}(a y)-\frac{P R}{2} \frac{\left(y-y^{2}\right)}{s}+\frac{P\left(1+s R \lambda_{2}\right)}{s^{2}\left(s \lambda_{1}+1\right)}-\frac{P R \lambda_{2}}{s\left(s \lambda_{1}+1\right)}$

Now the transformed boundary conditions are

$\bar{u}=\left\{\begin{array}{l}\frac{1}{s} ; y=1 \\ 0 ; y=0\end{array}\right.$

On solving equations (2.8) using (2.9), we

$$
\begin{aligned}
\bar{u}(y, s) & =\frac{P\left(1+s R \lambda_{2}\right)}{s^{2}\left(s \lambda_{1}+1\right)} \frac{\operatorname{Sinh}[a(1-y)]}{\operatorname{Sinh}(a)}+\frac{P R \lambda_{2}}{s\left(s \lambda_{1}+1\right)} \frac{\operatorname{Sinh}[a(1-y)]}{\operatorname{Sinh}(a)}+\frac{\operatorname{Sinh}(a y)}{\operatorname{Sinh}(a)} \\
& +\frac{P\left(1+s R \lambda_{2}\right)}{s^{2}\left(s \lambda_{1}+1\right)} \frac{\operatorname{Sinh}(a y)}{\operatorname{Sinh}(a)}+\frac{P R \lambda_{2}}{s\left(s \lambda_{1}+1\right)} \frac{\operatorname{Sinh}(a y)}{\operatorname{Sinh}(a)}-\frac{P R}{2} \frac{\left(y-y^{2}\right)}{s}-\frac{P\left(1+s R \lambda_{2}\right)}{s^{2}\left(s \lambda_{1}+1\right)}-\frac{P R \lambda_{2}}{s\left(s \lambda_{1}+1\right)}
\end{aligned}
$$

Taking the inverse Laplace transform for the equation (2.10); we get

$$
\begin{aligned}
u(y, t) & =y-\frac{P R}{2}\left(y-y^{2}\right)+\sum_{n=1}^{\infty}(-1)^{n} e^{s_{n_{1}} t}\left\{a_{13} \operatorname{Sinh}\left[b_{1}(1-y)\right]+a_{15} \operatorname{Sinh}\left(b_{1} y\right)\right\} \\
& +\sum_{n=1}^{\infty}(-1)^{n} e^{s_{n_{2}} t}\left\{a_{14} \operatorname{Sinh}\left[b_{2}(1-y)\right]+a_{16} \operatorname{Sinh}\left(b_{2} y\right)\right\}
\end{aligned}
$$

The shear stresses on the upper plate and the lower plate are given by 


$$
\begin{aligned}
& \tau_{L}=\left(\frac{d u}{d y}\right)_{y=0}=1-\frac{P R}{2}+\sum_{n=1}^{\infty}(-1)^{n} e^{s_{m} t}\left\{c_{2}-c_{1} \operatorname{Cosh}\left(b_{1}\right)\right\}+\sum_{n=1}^{\infty}(-1)^{n} e^{s_{n_{2}} t}\left\{c_{4}-c_{3} \operatorname{Cosh}\left(b_{2}\right)\right\} \\
& \tau_{U}=\left(\frac{d u}{d y}\right)_{y=1}=1+\frac{P R}{2}+\sum_{n=1}^{\infty}(-1)^{n} e^{s_{m_{m}} t}\left\{c_{2} \operatorname{Cosh}\left(b_{1}\right)-c_{1}\right\}+\sum_{n=1}^{\infty}(-1)^{n} e^{s_{n} t}\left\{c_{4} \operatorname{Cosh}\left(b_{2}\right)-c_{3}\right\}
\end{aligned}
$$

The mass flux $Q$ is given by

$$
Q=\int_{y=0}^{1} u d y=\frac{1}{2}-\frac{P R}{12}+\sum_{n=1}^{\infty}(-1)^{n} e^{s_{n 1} t} d_{5}\left[\operatorname{Cosh}\left(b_{1}\right)-1\right]+\sum_{n=1}^{\infty}(-1)^{n} e^{s_{n_{2}} t} d_{6}\left[\operatorname{Cosh}\left(b_{2}\right)-1\right]
$$

\section{Results and Discussion}

An initial boundary value problem is solved for the motion of an incompressible electrically conducting Oldroyd-B fluid confined in a channel bounded by two rigid non-conducting parallel plates and initially induced by a constant pressure gradient. The pressure gradient is suddenly withdrawn and the upper plate moves with a uniform velocity while the lower plate continues to be at rest. The velocity, the stresses and discharge between the plates are evaluated analytically with the help of Laplace transform technique and computationally discussed for different variations in the governing parameters. Hence, the flow governed by the non-dimensional parameters namely, $R$ the Reynolds number, $\lambda_{1}$ and $\lambda_{2}$ the Oldroyd-B fluid parameters, pressure $P$ and time $t$. Figures (2-8) represent the behaviour of the velocity component $u$ for variations in Reynolds number, material parameters, pressure and time $t$. Tables (1) shown that stresses on both boundaries and mass flux for different variations in parameters.

We noticed from the Figure (2-3), the magnitude of the velocity enhances with increasing Reynolds number R. The Reynolds number is a dimensionless quantity that is used to help predict similar flow patterns in different fluid flow situations. The Reynolds number is defined as the ratio of inertial forces to viscous forces and consequently quantifies the relative importance of these two types of forces for given flow conditions. Reynolds numbers frequently arise when performing scaling of fluid dynamics problems, and as such can be used to determine dynamic similitude between two different cases of fluid flow. They are also used to characterize different flow regimes within a similar fluid, such as laminar or turbulent flow: laminar flow occurs at low Reynolds numbers, where viscous forces are dominant, and is characterized by smooth, constant fluid motion; turbulent flow occurs at high Reynolds numbers and is dominated by inertial forces, which tend to produce chaotic eddies, vortices and other flow instabilities. For the both cases $(R=2$ to $10 \& 250$ to 1000) we observed that the velocity raise for the run up flow throughout the fluid region.

It is to be noticed that the velocity increases with increasing both the material parameters $\lambda_{1}$ and $\lambda_{2}$ (Figures 4-5). Moreover from Figure (6) depicts, the velocity is retarding with increasing the pressure prevails on the flow field. Consequently, it appears from Figures $(7 \& 8)$, the magnitude of the velocity component $u$ enhances with increasing values of time period. These provided a comparison how the increasing effects produced by different values of $R$ on the developing flow with the increase of time $t$.

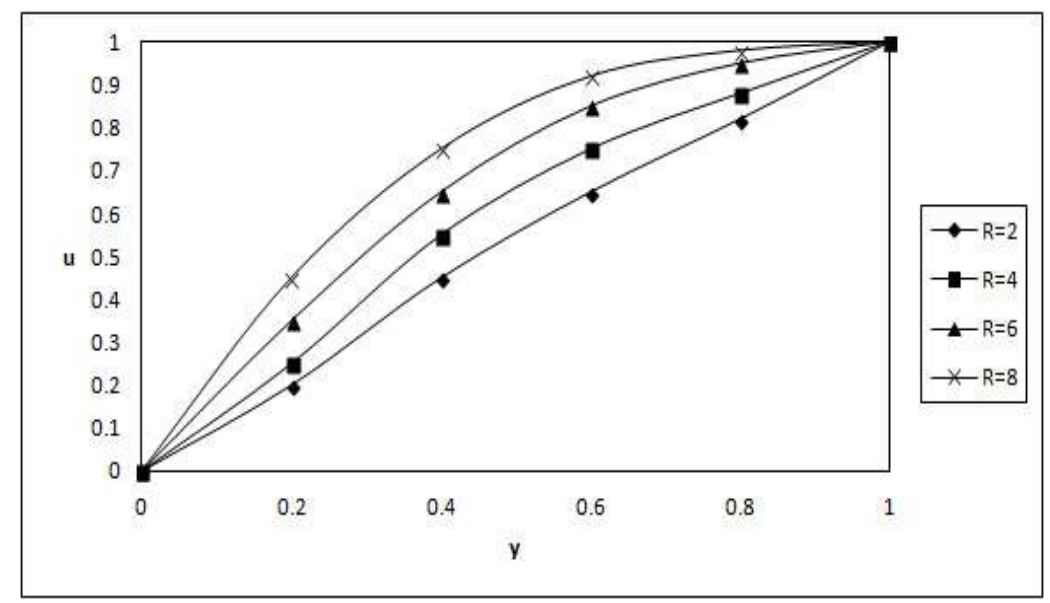

Fig. 2 The velocity profile for $u$ on Reynolds number $R$ $P=1, t=0.1, \lambda_{1}=5, \lambda_{2}=1$ 


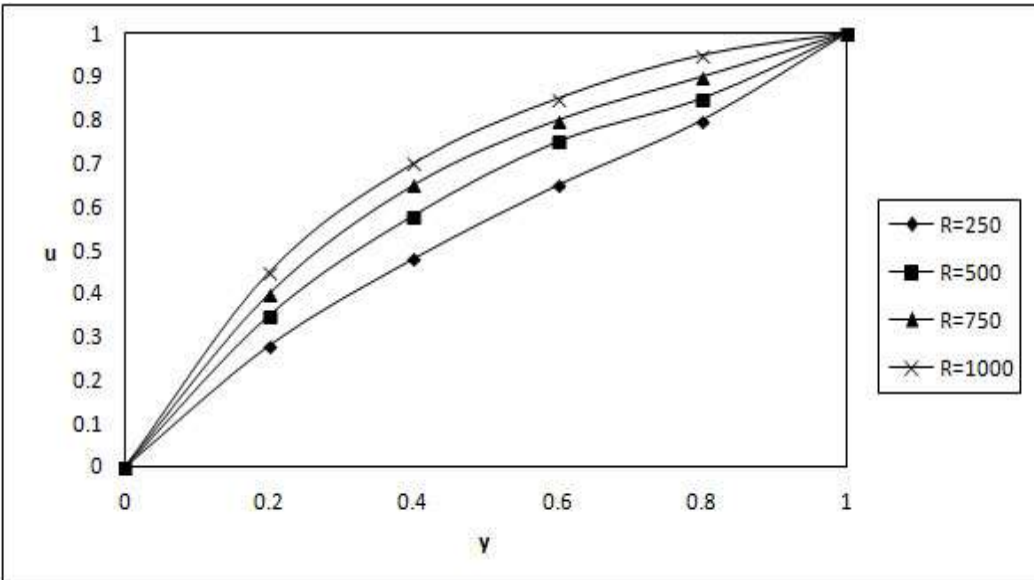

Fig. 3: The velocity profile for $u$ on Reynolds number $R$

$$
P=1, t=0.1, \lambda_{1}=5, \lambda_{2}=1
$$

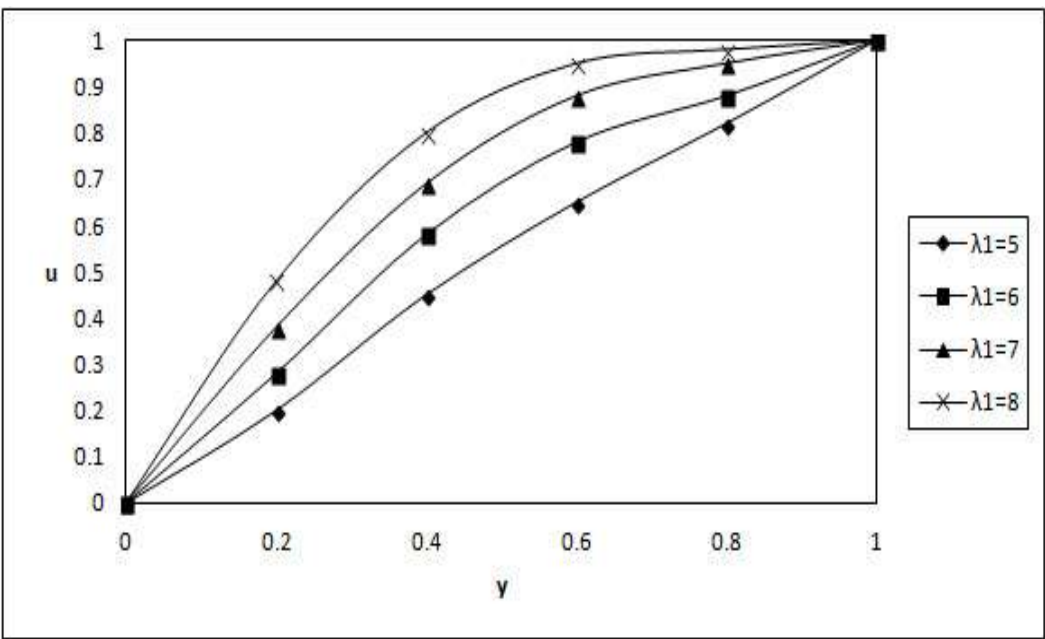

Fig. 4: The velocity profile for $u$ on the material parameter $\lambda_{1}$

$$
R=2, P=1, t=0.1, \lambda_{2}=1
$$

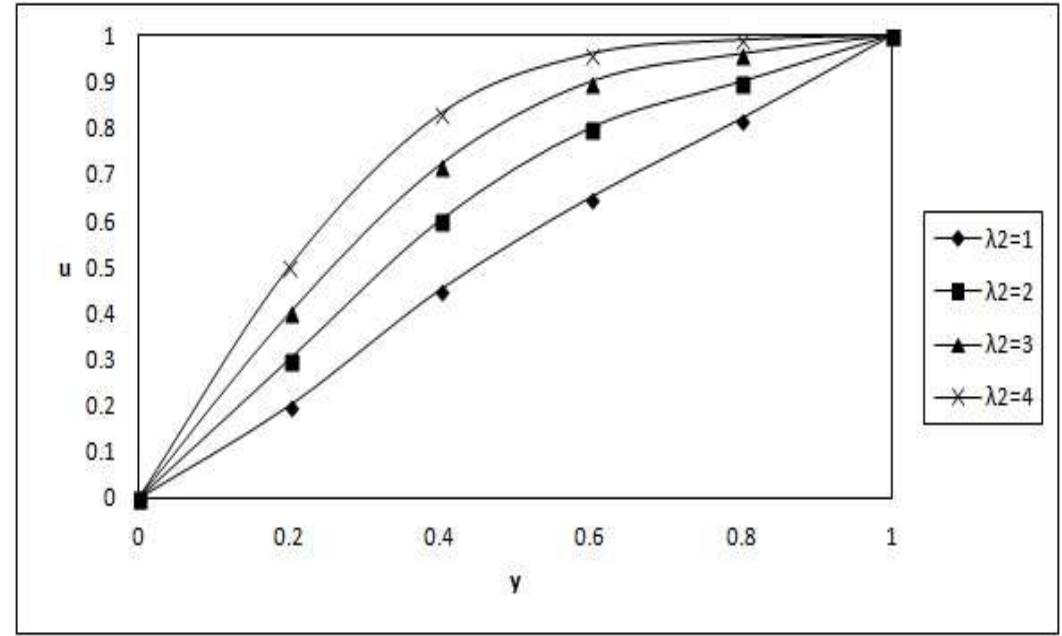

Fig. 5: The velocity profile for $u$ on the material parameter $\lambda_{2}$

$$
R=2, P=1, t=0.1, \lambda_{1}=5
$$




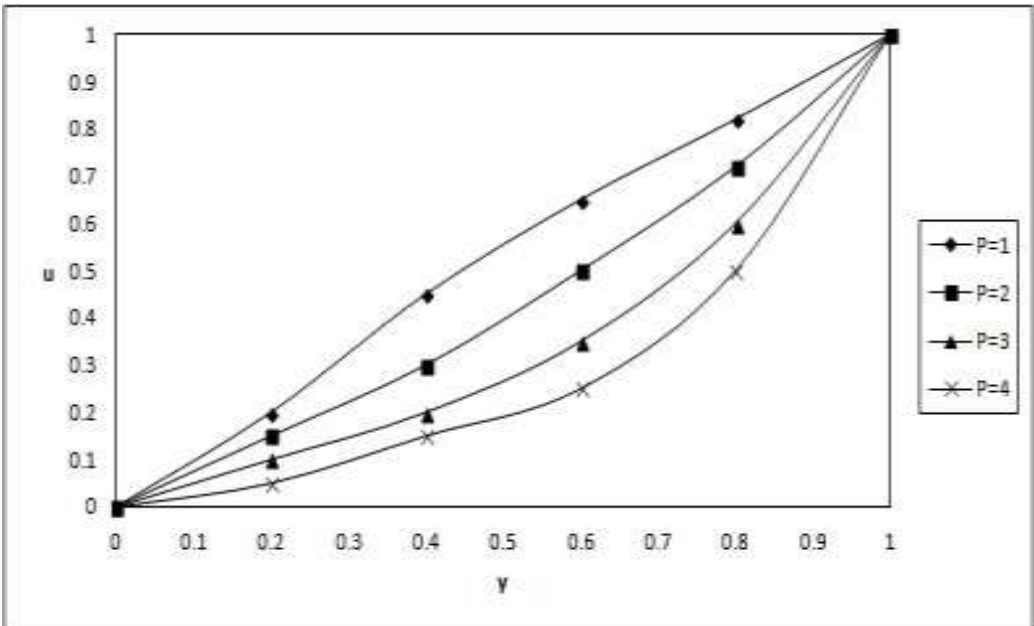

Fig. 6: The velocity profile for $u$ with pressure gradient $P$ $R=2, \lambda_{1}=5, \lambda_{2}=1, t=0.1$

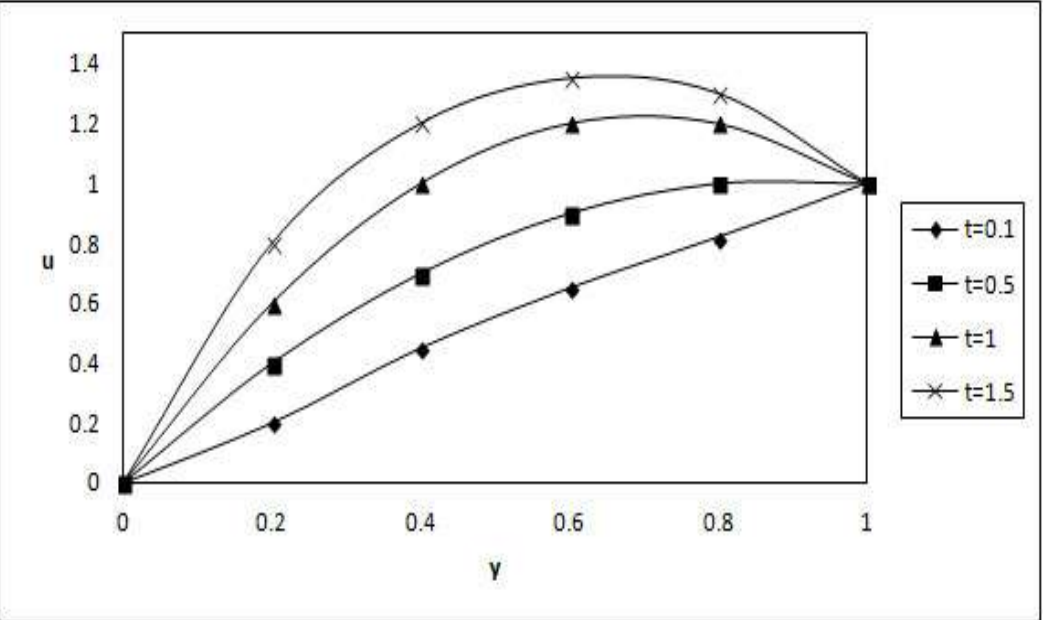

Fig. 7: Time development of the velocity component $u$

$$
R=2, P=1 \lambda_{1}=5, \lambda_{2}=1
$$

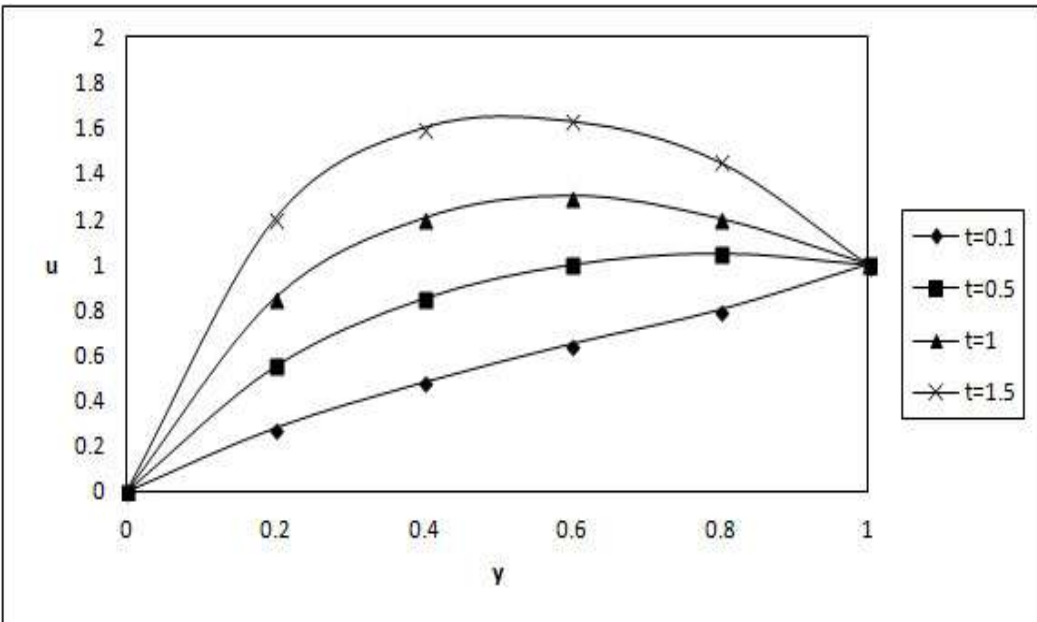

Fig. 8: Time development of the velocity component $u$

$$
R=1000, P=1, \lambda_{1}=5, \lambda_{2}=1
$$


The fluctuations of the skin friction (shear stresses) and mass flux with time on the lower and the upper plate are shown in the table (1) for different arbitrary non-zero values of $R, P, t, \lambda_{1}$ and $\lambda_{2}$. It is noticed that the skin friction increases with $R, \lambda_{1}, \lambda_{2}$ and $t$. at the upper plate and lower plates and diminishes with increasing $P$ at the same. It appears from this table that the skin-friction at the lower plate due to run up flow of Oldroyd-B fluid remains always less than its classical value while at the upper plate no such definite conclusion can be made. Also the similar behaviour is observed for the mass flux or discharge between the plates.

Table 1: The shear stresses and Mass flux

\begin{tabular}{cccccccc}
\hline$\lambda_{1}$ & $\lambda_{2}$ & $R$ & $P$ & $t$ & $\tau_{L}$ & $\tau_{U}$ & $Q$ \\
\hline $\mathbf{5}$ & $\mathbf{1}$ & $\mathbf{2}$ & $\mathbf{1}$ & $\mathbf{0 . 1}$ & 0.245262 & 0.585479 & 1.254420 \\
$\mathbf{6}$ & 1 & 2 & 1 & 0.1 & 0.286695 & 0.698804 & 5.785549 \\
$\mathbf{7}$ & 1 & 2 & 1 & 0.1 & 0.310558 & 0.877485 & 8.477008 \\
5 & $\mathbf{2}$ & 2 & 1 & 0.1 & 0.314452 & 0.715263 & 6.365853 \\
5 & $\mathbf{3}$ & 2 & 1 & 0.1 & 0.358879 & 1.255663 & 9.002545 \\
5 & 1 & $\mathbf{4}$ & 1 & 0.1 & 0.541206 & 0.988745 & 2.336524 \\
5 & 1 & $\mathbf{6}$ & 1 & 0.1 & 0.854665 & 1.522462 & 3.650086 \\
5 & 1 & 2 & $\mathbf{3}$ & 0.1 & 0.145226 & 0.211450 & 1.012232 \\
5 & 1 & 2 & $\mathbf{5}$ & 0.1 & 0.085471 & 0.145266 & 0.544856 \\
5 & 1 & 2 & 1 & $\mathbf{0 . 2}$ & 0.369961 & 0.966524 & 4.580476 \\
5 & 1 & 2 & 1 & $\mathbf{0 . 3}$ & 0.415262 & 1.566302 & 5.990145 \\
\hline
\end{tabular}

\section{Conclusions}

The run-up flow of an incompressible Oldroyd-B fluid between two parallel plates is studied. Analytical expressions for the fluid velocity field is obtained making use of Laplace transform domain. A similar type of problem in which the upper plate started impulsively from rest with velocity tooth pulses subjected on the upper plate with the lower plate kept stationary has already been solved in Ghosh et al. [22].

1. The magnitude of the velocity enhances with increasing Reynolds number, both material parameters as well as time.

2. When pressure increases the velocity diminishes throughout the fluid region.

3. Both the stresses raise with Reynolds number, both material parameters and trim down with pressure.

4. Mass flux reduces with pressure and develops with Reynolds number, material parameters and time.

\section{References}

[1] Naduvinamani. N.B., P.S. Hiremath, G. Gurubasavaraj, Squeeze film lubrication of a short porous journal bearing with couple stress fluids, Tribol. Int., 34(11), pp. 739-747, 2001.

[2] Naduvinamani. N.B., P.S. Hiremath, G. Gurubasavaraj, Surface roughness effects in a short porous journal bearing with a couple stress fluid, Fluid Dyn. Res., 31(5-6), pp. 333-354, 2002.

[3] Naduvinamani. N.B., P.S. Hiremath, G. Gurubasavaraj, Effects of surface roughness on the couple stress squeeze film between a sphere and a flat Plate, Tribol. Int., 38(5), pp. 451-458, 2005.

[4] Naduvinamani. N.B., Syeda Tasneem Fathima, P.S. Hiremath, Hydrodynamic lubrication of rough slider bearings with couple stress fluids, Tribol. Int., 36(12), pp. 949-959, 2003.

[5] Naduvinamani N.B., Syeda Tasneem Fathima, P.S. Hiremath, Effect of surface roughness on characteristics of couplestress squeeze film between anisotropic porous rectangular plates, Fluid Dyn. Res., 32(5), pp. 217-231, 2003.

[6] J.R. Lin, C.-R. Hung, Combined effects of non-Newtonian couple stresses and fluid inertia on the squeeze film characteristics between a long cylinder and an infinite plate, Fluid Dyn. Res., 39(8), pp. 616-639, 2007.

[7] J.Y. Kazakia, R.S. Rivlin, Run-up and spin-up in a viscoelastic fluid I, Rheol. Acta, 20(2), pp. 111-127, 1981.

[8] R.S. Rivlin, Run-up and spin-up in a viscoelastic fluid II, Rheol. Acta, 21(2), pp. 107-111, 1982.

[9] R.S. Rivlin, Run-up and spin-up in a viscoelastic fluid III, Rheol. Acta, 21(3), pp. 213-222, 1982.

[10] R.S. Rivlin, Run-up and spin-up in a viscoelastic fluid-IV, Rheol. Acta, 22(3), pp. 275-283, 1983.

[11] N.Ch. Pattabhi Ramacharyulu, K. Appala Raju, Run-up in a generalized porous medium, Indian J. Pure Appl. Math., 15(6), pp. 665-670, 1984.

[12] D. Ramakrishna, Some Problems in the Dynamics of Fluids with Particle Suspensions, Ph.D thesis, Kakatiya University, Warangal, India, 1986.

[13] G. Honig, U. Hirdes, A method for the numerical inversion of Laplace transforms, J. Comput. Appl. Math., 10(1), pp. 113-132, 1984.

[14] Diwakar.M and T.K.V. Iyengar, Run up flow of a couple stress fluid between parallel plates, Nonlinear Analysis: Modelling and Control, 15(1), pp. 29-37, 2010.

[15] Sugunamma.V, M. Sneha Latha and N. Sandeep, Run-up flow of a Rivlin-Ericksen fluid through a porous medium in a channel, International Journal of Mathematical Archive, 2(12), pp. 2625-2639, 2011.

[16] Veera Krishna.M, S.V.Suneetha and . R.Siva Prasad, "Hall current effects on unsteady MHD flow of rotating Maxwell fluid through a porous medium", Journal of Ultra Scientist of physical sciences, 22(1)M, pp.133-144, 2010.

[17] Raji Reddy Sheelam and Samba Siva Rao, "Computational Techniques in transient Magneto hydro dynamics Dusty viscous and Run up flows,", Ph.D. thesis, Osmania University, Hyderabad, Andhra Pradesh, India. pp. 47-65, 1992. 
[18] Raji Reddy.S, "Numerical Solution of Run-Up Flow through a Pipe with an Equilateral Triangular Cross-Section,” The IUP Journal of Computational Mathematics, 5(1), pp. 67-74, 2012

[19] Mahaboob Basha, "Visco-elastic fluid flow and the Heat Transfer through porous medium", Ph.D. thesis, S.K.University, Anantapur, Andhra Pradesh, India, 2005.

[20] Malleswari.D., "Usteady flow of a Rivlin-Ericksen fluid through plannar channels with porous lining", Ph.D., thesis, Sri Padmavathi Mahila Viswavidyalayam, Tirupati, Andhra Pradesh, India, 2010.

[21] M.Devakar and T.K.V.Iyengar, "Run up flow of an incompressible micropolar fluid between parallel plates - A state space approach," Applied Mathematical Modelling, 35(4), pp. 1751-1764, 2011. http://dx.doi.org/10.1016/j.apm.2010. 10.007.

[22] A.K. Ghosh and P. Sana, "On hydromagnetic channel flow of an Oldroyd-B fluid induced by tooth pulses," Magnetohydro dynamics, 44(3), pp. 325-340, 2008.

\section{Appendix:}

$$
\begin{aligned}
& a=\sqrt{\frac{R\left(s^{2} \lambda_{1}+s\right)}{1+s R \lambda_{2}}} ; b=R+n^{2} R \lambda_{2} \pi^{2} ; c=n^{2} \pi^{2} ; s_{n_{1}}=\frac{-b+\sqrt{b^{2}-4 R \lambda_{1} c}}{2 R \lambda_{1}} ; s_{n_{1}}=\frac{-b+\sqrt{b^{2}-4 R \lambda_{1} c}}{2 R \lambda_{1}} ; \\
& s_{n_{2}}=\frac{-b-\sqrt{b^{2}-4 R \lambda_{1} c}}{2 R \lambda_{1}} ; a_{1}=\frac{\sqrt{R}}{2\left(1+s_{n_{1}} R \lambda_{2}\right)}\left\{\frac{\left(1+s_{n_{1}} R \lambda_{2}\right)\left(1+2 s_{n_{1}} \lambda_{1}\right)-R \lambda_{2}\left(s_{n_{1}}+s_{n_{1}}{ }^{2} \lambda_{1}\right)}{\sqrt{s_{n_{1}}+s_{n_{1}}{ }^{2} \lambda_{1}} \sqrt{1+s_{n_{1}} R \lambda_{2}}}\right\} ; \\
& a_{2}=\frac{P\left(1+s_{n_{1}} R \lambda_{2}\right)}{s_{n_{1}}^{2}\left(1+s_{n_{1}} \lambda_{1}\right)} ; \quad a_{3}=\frac{a_{2}}{a_{1} \operatorname{Cosh}\left(b_{1}\right)} ; \\
& a_{4}=\frac{\sqrt{R}}{2\left(1+s_{n_{2}} R \lambda_{2}\right)}\left\{\frac{\left(1+s_{n_{2}} R \lambda_{2}\right)\left(1+2 s_{n_{2}} \lambda_{1}\right)-R \lambda_{2}\left(s_{n_{2}}+s_{n_{2}}{ }^{2} \lambda_{1}\right)}{\sqrt{s_{n_{2}}+s_{n_{2}}{ }^{2} \lambda_{1}} \sqrt{1+s_{n_{2}} R \lambda_{2}}}\right\} \text {; } \\
& a_{5}=\frac{P\left(1+s_{n_{2}} R \lambda_{2}\right)}{s_{n_{2}}{ }^{2}\left(1+s_{n_{2}} \lambda_{1}\right)} ; a_{6}=\frac{a_{5}}{a_{4} \operatorname{Cosh}\left(b_{2}\right)} ; a_{7}=\frac{P R \lambda_{2}}{s_{n_{1}}\left(1+s_{n_{1}} \lambda_{1}\right)} ; a_{8}=\frac{a_{7}}{a_{1} \operatorname{Cosh}\left(b_{1}\right)} ; \\
& a_{9}=\frac{P R \lambda_{2}}{s_{n_{2}}\left(1+s_{n_{2}} \lambda_{1}\right)} ; a_{10}=\frac{a_{9}}{a_{4} \operatorname{Cosh}\left(b_{2}\right)} ; a_{11}=\frac{1}{a_{1} s_{n_{1}} \operatorname{Cosh}\left(b_{1}\right)} ; a_{12}=\frac{1}{a_{4} s_{n_{2}} \operatorname{Cosh}\left(b_{2}\right)} ; \\
& a_{13}=a_{3}+a_{8} ; a_{15}=a_{11}+a_{3}+a_{8} ; a_{14}=a_{6}+a_{10} ; a_{16}=a_{12}+a_{6}+a_{10} ; \\
& b_{1}=\sqrt{\frac{R\left(s_{n_{1}}{ }^{2} \lambda_{1}+s_{n_{1}}\right)}{1+s_{n_{1}} R \lambda_{2}}}, \quad b_{2}=\sqrt{\frac{R\left(s_{n_{2}}{ }^{2} \lambda_{1}+s_{n_{2}}\right)}{1+s_{n_{2}} R \lambda_{2}}} ; \\
& c_{1}=a_{13} b_{1} ; \quad c_{2}=a_{15} b_{1} ; \quad c_{3}=a_{14} b_{2} ; c_{4}=a_{16} b_{2} \text {; } \\
& d_{1}=\frac{a_{13}}{b_{1}} ; \quad d_{2}=\frac{a_{15}}{b_{1}} ; \quad d_{3}=\frac{a_{14}}{b_{2}} ; d_{4}=\frac{a_{16}}{b_{2}} ; d_{5}=d_{1}+d_{2} ; d_{6}=d_{3}+d_{4} .
\end{aligned}
$$

